\title{
A REMARK ON A RESULT OF MARVIN MARCUS
}

A. L. Dulmage and N. S. Mendelsohn*

(received September 24, 1962)

Marcus [2] has proved the following theorem.

Suppose $A$ is a non-negative normal matrix satisfying $\mathrm{p}(\mathrm{A})=0$ in which $\mathrm{p}(\lambda)$ is a monic polynomial no two of whose non-zero roots have the same modulus. Then the re exists a permutation matrix $\mathrm{P}$ such that $\mathrm{PAP}^{*}$ is a direct sum, $\operatorname{PAP}^{*}=\mathrm{A}_{1} \oplus \mathrm{A}_{2} \oplus \ldots \oplus \mathrm{A}_{\mathrm{m}}$, in which each $\mathrm{A}_{\mathrm{i}}$ is eithe $\mathrm{r}$ O or primitive.

This note gives a gene ralisation of this result, dropping the non-negative assumption and weakening the normality as sumption.

Remark 1. If $\mathrm{A}$ is an $\mathrm{n}$ by $\mathrm{n}$ matrix whose elements a re real and if $\mathrm{AA}^{\mathrm{T}}$ and $\mathrm{A}^{\mathrm{T}} \mathrm{A}$ have the same diagonal elements then there exists a permutation matrix $P$ such that $P^{-1} A P=$ $\operatorname{diag}\left(A_{1}, A_{2}, \ldots, A_{m}\right)$ in which the $A_{i}$ are irreducible.

Proof: There exists a permutation matrix $P$ such that

$$
P^{-1} A P=\left[\begin{array}{cccc}
A_{11} & 0 & \cdots & 0 \\
A_{21} & A_{22} & \cdots & 0 \\
\cdot & \cdot & & \cdot \\
A_{m 1} & & & A_{\mathrm{mm}}
\end{array}\right]
$$

* This research was supported by the United States Air Force Office of Scientific Research.

Canad. Math. Bull. vol. 6, no. 1, Janua ry 1963. 
where $A_{11}, A_{22}, \ldots, A_{m m}$ are irreducible.

Let $A_{11}$ be a $t$ by $t$ matrix. Since $A A^{T}$ and $A^{T} A$ have the same diagonal elements it follows that the sum of the squares of the elements in row $i$ of $A$ is equal to the sum of the squares of the elements in column $i$ of $A$, for $i=1,2, \ldots, n$. The same is true of $P^{-1} A P$. Also, the sum of the squares of the elements in the first $t$ rows of $P^{-1} A P$ is equal to the sum of the squares of the elements of $A_{11}$ which in turn is equal to the sum of the squares of the elements of the first $t$ columns of $P^{-1} A P$. It follows that $A_{21}, A_{31}$, $\ldots, \mathrm{A}_{\mathrm{m} 1}$ have all their elements equal to 0 .

Similar remarks relative to $\mathrm{A}_{22}$ show that $\mathrm{A}_{32}, \mathrm{~A}_{42}$, $\ldots, A_{\mathrm{m} 2}$ have all their entries equal to 0 . Repeating the argument we have $A_{u v}=0$ for $u \neq v$. Put $A_{i}=A_{i i}$ and Remark 1 is proved.

The directed graph $D_{A}$ of an $n$-square matrix $A$ is defined as follows. It has vertex set $(1,2, \ldots, n)$, and the ordered pair $(i, j)$ is an edge of $D_{A}$ if and only if $A_{i j} \neq 0$. A directed graph $D$ with vertex set $V$ is cyclically k-partite $(\mathrm{k} \geq 2)$, if and only if $\mathrm{V}$ can be partitioned, $\mathrm{V}=\mathrm{V}_{1}+\mathrm{V}_{2}+$ $\ldots+V_{k}$ such that $(i, j)$ is an edge of $D$ only if $i \in V_{1}$ and $j \in V_{2}$, or $i \in V_{2}$ and $j \in V_{3}$, or ... or $i \in V_{k}$ and $j \in V_{1}$.

It has been remarked [1] that if $D_{A}$ is cyclically $k$-partite then the characteristic polynomial of $A$ has the form $f\left(\lambda^{k}\right) \lambda^{p}$. Remark 2 and Remark 3 follow from this observation.

Remark 2. If $D_{A}$ is cyclically $k$-partite $(k \geq 2)$, then for every non-zero characteristic root $\lambda$ of $A$ there exist at lea st k-1 distinct roots which a re distinct from $\lambda$ and have the same modulus as $\lambda$. 
Remark 3. If the minimal polynomial of a matrix $A$ has a root $\lambda \neq 0$ and has no root $\mu$ such that $\mu \neq \lambda$ and $|\lambda|=|\mu|$, then there is no integer $k \geq 2$ for which $D_{A}$ is cyclically $\mathrm{k}$-partite.

Remark 1 and Remark 3 give us the following theorem.

THEOREM 1. Let $A$ be an $n$ by $n$ matrix with real elements such that $A A^{T}$ and $A^{T} A$ have the same diagonal elements. Suppose $\mathrm{p}(\lambda)$ is a monic polynomial such that (i) $\mathrm{p}(\mathrm{A})=0$, and (ii) $\mathrm{p}(\lambda)$ has no pair of roots $\lambda$ and $\mu$ with $\lambda \neq \mu$ and $|\lambda|=|\mu|$. Then the re exists a permutation matrix $P$ such that $P^{-1} A P=\operatorname{diag}\left[A_{1}, A_{2}, \ldots, A_{m}\right]$ in which the matrices $A_{i}$ are irreducible. Moreover, for each $A_{i}$, either every root is zero or there exists no integer $k \geq 2$ such that the directed graph $\mathrm{D}_{\mathrm{A}_{i}}$ is cyclically $\mathrm{k}$-partite.

In the result of Marcus [2] the assumption that $A$ is nonnegative implies that an $A_{i}$ which is not zero has a non-zero characteristic root $\lambda$ and thus the first alternative that every root of $A_{i}$ should be zero is not possible. If $A_{i}$ is imprimitive then $D_{A}$ is cyclically d-partite where $d$ is the index of imprimitivity. Thus the second alternative, that the re is no $k \geq 2$ such that $D_{A_{i}}$ is cyclically $k$-partite, implies that $A_{i}$

is primitive. Thus Theorem 1 generalizes Marcus' Theorem.

\section{REFERENCES}

1. A. L. Dulmage and N.S. Mendelsohn "The Characteristic Equation of an Imprimitive Matrix". Submitted to the Journal of S. I. A. M.

$\therefore \quad$ Marvin Marcus "Another Remark on a Result of K. Goldberg". Can. Math. Bull. 6 (1963). p. 7. 